Revista Colombiana de Obstetricia y Ginecología Vol. 58 No. 2 • $2007 \bullet(136-141)$

Artículo de Revisión

\title{
PANORAMA ACTUAL DE LOS INHIBIDORES DE LA AROMATASA EN LA INDUCCIÓN DE LA OVULACIÓN
}

\author{
Current view of aromatase inhibitors \\ in ovulation induction \\ Juan de Jesús Pachón-Rincón, M.D.*, Natalia Posada-Villa, M.D.** \\ Recibido: junio 8/06 - Revisado: agosto 9/06 - Respuesta autores: abril 18/07 - Aceptado: mayo 18/07
}

\section{RESUMEN}

El panorama actual de la inducción de la ovulación en el tratamiento de la infertilidad está cambiando debido a la introducción reciente en este campo de los inhibidores de la aromatasa de tercera generación (IA). Estos agentes han mostrado su eficacia como inductores de la ovulación, mediante la inhibición de la aromatasa que convierte los andrógenos en estrógenos, necesarios para el desarrollo y la maduración folicular. Este mecanismo de acción hace a los IA ventajosos frente al citrato de clomifeno, por carecer de efectos antiestrogénicos sobre el endometrio y el moco cervical. Presentamos una revisión de los tópicos más importantes con base en estudios publicados acerca del uso clínico de los IA.

Palabras clave: inhibidores de la aromatasa, inducción de la ovulación, citrato de clomifeno, gonadotropinas, endometrio, embarazo, aborto.

\section{SUMMARY}

The current overall view regarding ovulation induction in infertility treatment is changing because of the recent introduction of third-generation aromatase inhibitors in this area. These agents have been shown to be effective as ovulation inductors because they inhibit

\footnotetext{
* Especialista en ginecología y obstetricia. Universidad de Antioquia. Medellín (Colombia). Correo electrónico: jpachon4@gmail.com

** Ginecóloga y obstetra, especialista en medicina reproductiva. Instituto Antioqueño de Reproducción (INSER). Calle 12 No 39-60, Medellín (Colombia)
}

aromatase, the enzyme that converts androgens into estrogens, a necessary step for follicular development and maturation. Special emphasis has been placed on the mechanism of action of the aromatase inhibitor compared to that of clomiphene citrate, since the aromatase inhibitor lacks an anti-oestrogenic effect on the endometrium and cervical mucus. This is a review of the most recent evidence supporting the use of aromatase inhibitors as ovulation-inductors based on currently published studies.

Key words: aromatase inhibitors, ovulation induction, clomiphene citrate, gonadotropins, endometrium, pregnancy, miscarriage.

\section{Definición y aspectos históricos}

Los inhibidores de la aromatasa (IA) son agentes no esteroideos que suprimen la biosíntesis de estrógenos por inhibición reversible de la enzima que convierte andrógenos en estrógenos. De este modo se reduce la retroalimentación negativa sobre el sistema hipotálamo-hipófisis, lo cual resulta en un incremento de la secreción de la hormona folículo estimulante (FSH, por su sigla en inglés) por parte de la hipófisis, que puede llevar a la selección y maduración de los folículos ováricos. ${ }^{1}$ Aunque la vía final común de los IA y el citrato de clomifeno es la misma, el mecanismo de acción es claramente diferente y esto le podría conferir ventajas a los inhibidores en el terreno de la inducción de la ovulación. ${ }^{2}$ 
Los IA se han usado ampliamente en el tratamiento del cáncer de mama. La primera generación de estos agentes apareció en la década del 60, pero sus efectos secundarios limitaron su uso. ${ }^{3}$ Con la aparición de los IA de tercera generación se ha dado un paso importante debido a la alta potencia y pocos efectos secundarios conocidos de estas nuevas drogas. Estos agentes se usan cada vez más en protocolos de estimulación ovárica, con buenos resultados. ${ }^{2}$

\section{Características farmacológicas de los inhibidores de la aromatasa}

Los IA de tercera generación se dividen en dos grupos: los inhibidores competitivos, los cuales se unen al sitio activo de la enzima, y los inactivadores, que destruyen la enzima al unirse covalentemente a ella. Letrozole y anastrozole son inhibidores competitivos y exemestane es inactivador. ${ }^{3,4}$

La vida media del anastrozole a dosis de $1 \mathrm{mg}$ diario es de 41-48 horas y la de letrozole a dosis de 2,5 mg al día es de 2-4 días. ${ }^{3}$ En sistemas celulares letrozole es 10-20 veces más potente que anastrozole en cuanto a la inhibición de aromatasa. ${ }^{5}$ A dosis de 1-5 mg por día los IA disminuyen los niveles de estrógenos entre un 97 y un 99\%.

El metabolismo se realiza principalmente en el hígado. Sus efectos adversos principales son alteraciones gastrointestinales, astenia, oleadas de calor, dolor de cabeza y de espalda. ${ }^{6}$

Se ha planteado que los IA pueden tener un rol estimulante en el crecimiento folicular temprano mediante el aumento de los receptores de FSH a nivel ovárico y por tanto amplificarían la acción de esta hormona. ${ }^{7,8}$

\section{Evaluación de diferentes inhibidores de la aromatasa en la inducción de la ovulación y definición de la dosis más apropiada}

Al-Omari y cols. trataron a 22 pacientes con síndrome de ovario poliquístico (SOP), resistentes al citrato de clomifeno, con 2,5 mg/día de letrozole y a 18 pacientes de características similares con $1 \mathrm{mg}$ diario de anastrozole, en un estudio aleatorio, doble ciego. Ambos medicamentos fueron administrados del día 3 al 7 del ciclo menstrual.

El grosor endometrial y el porcentaje de ovulación fueron significativamente mayores en el grupo de letrozole comparados con el grupo de anastrozole $(8,17$ vs. $6,53 \mathrm{~mm}$ y 84,4 vs. $60 \%, \mathrm{p}<0,05$, respectivamente). El porcentaje de embarazo clínico por ciclo también fue estadísticamente más alto en el grupo de letrozole comparado con anastrozole $(18,8$ vs. 9,7\%, $\mathrm{p}<0,05)$. El número promedio de folículos fue igual en los dos grupos.

No se presentaron embarazos múltiples. Los autores concluyeron que los IA son efectivos en la inducción de la ovulación y consecución de embarazo en mujeres anovulatorias con SOP resistentes a clomifeno. Se observaron mejores resultados con letrozole comparado con anastrozole. ${ }^{9}$

Al-Fadhli y cols. realizaron un estudio prospectivo aleatorio para evaluar el efecto de 2,5 vs. $5 \mathrm{mg}$ al día de letrozole durante los días 3 al 7 del ciclo para inducir superovulación en un total de 72 parejas sometidas a inseminación intrauterina. Treinta y cuatro mujeres recibieron $2,5 \mathrm{mg}$ y 38 mujeres, $5 \mathrm{mg}$. La ovulación fue inducida con 10.000 UI de gonadotropina coriónica humana (hCG).

El número total de folículos dominantes el día de la hCG, al igual que el porcentaje de embarazos por ciclo $(26,3 \%$ vs. $5,9 \%)$ fue significativamente mayor $(\mathrm{p}<0,05)$ en el grupo de $5 \mathrm{mg}$. No se presentaron embarazos múltiples en ninguno de los dos grupos. El grosor endometrial fue similar. Los autores concluyeron que $5 \mathrm{mg}$ diarios de letrozole durante 5 días parece ser la dosis más adecuada para producir superovulación. ${ }^{10}$

\section{Inhibidores de la aromatasa comparados con el citrato de clomifeno}

Hasta ahora el citrato de clomifeno ha sido el medicamento de primera elección para tratar la infertilidad de las pacientes en el grupo II de la Organización 
Mundial de la Salud donde la mayoría tienen SOP. Han surgido nuevas alternativas que pueden desplazar al clomifeno como primera línea de tratamiento en el SOP y estas comprenden la metformina, los IA y la FSH en dosis bajas. Esto podría representar el comienzo del fin de la era del clomifeno. ${ }^{2}$

Los IA se han comparado de manera directa con el clomifeno para evaluar su efecto sobre el desarrollo folicular y el perfil hormonal, con resultados favorables. ${ }^{11}$ Además, varios estudios han evaluado el papel de los IA en pacientes anovulatorias con SOP resistentes a clomifeno ${ }^{7}$ y en mujeres con infertilidad idiopática ${ }^{12}$, mostrando buenos resultados.

La percepción actual es que los IA pueden ser una buena alternativa frente al clomifeno para la inducción de la ovulación y la superovulación, pero se esperan estudios aleatorios más grandes que confirmen las ventajas clínicas de estos agentes. ${ }^{2}$

\section{Inhibidores de la}

\section{aromatasa en superovulación}

Un estudio prospectivo aleatorio que evaluó 238 ciclos de superovulación en mujeres con infertilidad idiopática, comparó el tratamiento con letrozole (7,5 $\mathrm{mg}$ /día) con el clomifeno (100 mg/día) por 5 días. No hubo diferencia significativa entre el grupo de letrozole y el de clomifeno en el grosor endometrial $(7,1 \mathrm{~mm}$ y 8,2 mm, IC 95\%: -1,1 a -0,2, respectivamente) ni en el porcentaje de embarazo por ciclo $(11,5 \%$ vs. $8,9 \%$, respectivamente). Se presentaron 4 abortos entre los 11 embarazos en el grupo de clomifeno, mientras que en el grupo de letrozole no se presentaron abortos aunque hubo dos embarazos ectópicos. ${ }^{13}$

\section{Inhibidores de la aromatasa en pacientes con SOP}

En un estudio de 44 pacientes con SOP resistente al clomifeno, Elnashar y cols. evaluaron la eficacia del letrozole en la inducción de la ovulación, comparando las características demográficas y clínicas de las mujeres que respondieron y que no respondieron a este agente. Todas las pacientes habían recibido 6 ciclos con dosis de hasta $150 \mathrm{mg}$ de clomifeno al día por 5 días, sin respuesta. Se les inició letrozole 2,5 mg/día del día 3 al 7 del ciclo menstrual, 2 meses después de haber recibido tratamiento con el clomifeno. Se programó el coito luego de la administración de 10.000 UI de hCG para desencadenar la ovulación.

La ovulación ocurrió en el 54,6\% de las pacientes durante el ciclo de tratamiento y el porcentaje de embarazo clínico fue de $25 \%$. No se presentaron embarazos múltiples ni abortos. Se compararon las pacientes que respondieron a letrozole $(\mathrm{n}=24)$ con las que no respondieron $(\mathrm{n}=20)$ en cuanto a la edad, duración de la infertilidad, índice de masa corporal, relación cintura/cadera, LH, FSH y relación LH/FSH, y no se encontraron diferencias significativas; por tanto, la inducción con letrozole no parece depender de variables como la obesidad o el perfil hormonal. ${ }^{14}$

Mitwally y Casper obtuvieron resultados similares en un estudio prospectivo utilizando letrozole como inductor de la ovulación en pacientes que no habían respondido a clomifeno. ${ }^{7}$

\section{Inhibidores de la aromatasa como coadyuvantes en los protocolos de estimulación ovárica con gonadotropinas}

En un estudio prospectivo no aleatorizado de 110 pacientes con infertilidad inexplicada, 36 de ellas recibieron tratamiento con letrozole y FSH; 18 con clomifeno y FSH y 56 con FSH sola. La FSH se inició entre los días 3 a 7 del ciclo en forma continua hasta el día de aplicación de la hCG para desencadenar la ovulación. Se requirió menor cantidad de gonadotropina en el grupo de letrozole y en el grupo de clomifeno comparado con el grupo de FSH sola. La cantidad de folículos dominantes fue similar en los tres grupos. El porcentaje de embarazo clínico por ciclo fue significativamente más bajo en el grupo de clomifeno con FSH (10,5\%) en comparación con el grupo de letrozole y FSH $(19,1 \%)$ y el grupo de FSH sola $18,7 \%, \mathrm{p}<0,05) .{ }^{15}$ 
Otros estudios han reportado resultados similares. ${ }^{6,16}$ Los IA han mostrado ser útiles como coadyuvantes en pacientes con pobre respuesta a las gonadotropinas. ${ }^{17}$ Los IA también parecen promisorios en el contexto de la fertilización in vitro. ${ }^{18}$ Por lo tanto se plantea que la adición de un IA a los protocolos de gonadotropinas disminuye los requerimientos de estas e incrementa el número de folículos preovulatorios sin producir un efecto negativo sobre el porcentaje de embarazos clínicos. ${ }^{19}$

\section{Resultados de embarazos luego de la estimulación de la ovulación con inhibidores de la aromatasa}

Mitwally y Casper recientemente, en un estudio no controlado, evaluaron los resultados de embarazos en una cohorte de mujeres después de la estimulación ovárica con letrozole, en comparación con otros tratamientos de estimulación ovárica incluidos clomifeno, gonadotropinas y un grupo control con embarazo espontáneo, sin estimulación ovárica.

Los grupos de mujeres con embarazo después de tratamiento y el grupo control eran similares en edad, duración y diagnóstico de infertilidad.

No hubo diferencia en el porcentaje de aborto y embarazo ectópico entre los diferentes grupos pero el letrozole estuvo asociado con un porcentaje de gestaciones múltiples significativamente inferior $(\mathrm{p}<0,05)$ comparado con el uso de clomifeno. ${ }^{20}$

\section{Teratogenicidad $y$ malformaciones congénitas}

No se dispone de estudios acerca de la teratogenicidad de los IA en humanos. Los estudios en animales (ratas y conejas) han mostrado que dosis bajas de letrozole producen efectos nocivos sobre el embrión. ${ }^{21}$ Sin embargo, se plantea que estos efectos se relacionan con la exposición continua a la sustancia durante la organogénesis y que después de la administración del medicamento, en la fase folicular temprana, este se ha eliminado casi totalmente para el momento de la ovulación, debido a su corta vida media. Se debe descartar el embarazo antes de iniciar letrozole. ${ }^{22}$ En España el laboratorio fabricante (Novartis) bajo la marca de Femara indica que este producto no está indicado en inducción de la ovulación, ya que se carece de estudios adecuados y bien controlados en humanos.

Por otro lado, la información disponible por parte del laboratorio fabricante de anastrozole bajo la marca de Arimidex (AstraZeneca) indica que la administración oral de anastrozole a ratas y conejas preñadas a dosis de hasta 1,0 y 0,2 $\mathrm{mg} / \mathrm{kg} /$ día, respectivamente, no causa efectos teratogénicos. ${ }^{23}$

En un informe reciente, Tulandi y cols. reportaron la incidencia de malformaciones congénitas en más de 900 niños concebidos después del tratamiento de infertilidad con letrozole o clomifeno. No se incluyeron nacimientos luego de fertilización in vitro. No hubo diferencia en la edad de las madres, peso al nacer y porcentaje de embarazos múltiples entre los dos grupos de estudio.

Las malformaciones congénitas y anormalidades cromosómicas se presentaron en el 2,4\% de los 514 niños nacidos luego del tratamiento con letrozole y en el 4,8\% de los 397 nacidos, luego del uso de clomifeno. Se notó una mayor frecuencia de malformaciones cardíacas en el grupo de clomifeno. Los autores concluyeron que no hubo diferencia en el índice global de malformaciones congénitas entre los dos grupos pero que aparentemente las anomalías cardíacas son menos frecuentes con el uso de letrozole comparado con clomifeno. ${ }^{24}$

\section{CONCLUSIÓN}

Los resultados de los estudios publicados hasta el momento sugieren que el panorama actual de los IA, especialmente de letrozole, como inductores de la ovulación es prometedor. Se destaca como una ventaja de los IA frente al clomifeno, la ausencia de un efecto antiestrogénico a nivel del endometrio y el moco cervical.

Aunque se ha planteado cierta preocupación debido a la carencia de estudios adecuados de teratogenicidad en humanos, la tendencia clínica es hacia 
el aprovechamiento del potencial terapéutico de estos agentes, para desplazar al clomifeno en varios escenarios de la reproducción asistida donde hasta ahora este se ha afianzado durante cuatro décadas, y del servicio que prestan como coadyuvantes en los protocolos de estimulación ovárica con gonadotropinas, con el fin de mejorar los resultados y disminuir los costos.

Se esperan resultados de estudios prospectivos bien diseñados, con una mayor muestra de pacientes que confirmen la eficacia y seguridad de los IA en la inducción de la ovulación.

\section{REFERENCIAS}

1. Messinis IE. Ovulation induction: a mini review. Hum Reprod 2005;20:2688-97.

2. Homburg R. Clomiphene citrate--end of an era? A mini review. Hum Reprod 2005;20:2043-51.

3. Buzdar AU, Robertson JF, Eiermann W, Nabholtz JM. An overview of the pharmacology and pharmacokinetics of the newer generation aromatase inhibitors anastrozole, letrozole, and exemestane. Cancer 2002;95:2006-16.

4. Santen RJ. Inhibition of aromatase: insights from recent studies. Steroids 2003;68:559-67.

5. Haynes BP, Dowsett M, Miller WR, Dixon JM, Bhatnagar AS. The pharmacology of letrozole. J Steroid Biochem Mol Biol 2003;87:35-45.

6. Holzer H, Casper R, Tulandi T. A new era in ovulation induction. Fertil Steril 2006;85:277-84.

7. Healey S, Tan SL, Tulandi T, Biljan MM. Effects of letrozole on superovulation with gonadotropins in women undergoing intrauterine insemination. Fertil Steril 2003;80:1325-9.

8. Mitwally MF, Casper RF. Use of an aromatase inhibitor for induction of ovulation in patients with an inadequate response to clomiphene citrate. Fertil Steril 2001;75:305-9.

9. Al-Omari WR, Sulaiman WR, Al-Hadithi N. Comparison of two aromatase inhibitors in women with clomiphene-resistant polycystic ovary syndrome. Int J Gynaecol Obstet 2004;85:289-91.

10. Al-Fadhli R, Sylvestre C, Buckett W, Tan SL, Tulandi T. A randomized trial of superovulation with two different doses of letrozole. Fertil Steril 2006;85:161-4.
11. Fisher SA, Reid RL, Van Vugt DA, Casper RF. A randomized double-blind comparison of the effects of clomiphene citrate and the aromatase inhibitor letrozole on ovulatory function in normal women. Fertil Steril 2002;78:280-5.

12. Mitwally MF, Casper RF. Aromatase inhibitors for the treatment of infertility. Expert Opin Investig Drugs 2003;12:353-71.

13. Al-Fozan H, Al-Khadouri M, Tan SL, Tulandi T. A randomized trial of letrozole versus clomiphene citrate in women undergoing superovulation. Fertil Steril 2004;82:1561-3.

14. Elnashar A, Fouad H, Eldosoky M, Saeid N. Letrozole induction of ovulation in women with clomiphene citrate-resistant polycystic ovary syndrome may not depend on the period of infertility, the body mass index, or the luteinizing hormone/follicle-stimulating hormone ratio. Fertil Steril 2006;85:511-3.

15. Mitwally MF, Casper RF. Aromatase inhibition reduces gonadotrophin dose required for controlled ovarian stimulation in women with unexplained infertility. Hum Reprod 2003;18:1588-97.

16. Mitwally MF, Casper RF. Aromatase inhibition reduces the dose of gonadotrophin required for controlled ovarian hyperstimulation. J Soc Gynecol Investig 2004;11:406-15.

17. Mitwally MF, Casper RF. Aromatase inhibition improves ovarian response to follicle-stimulating hormone in poor responders. Fertil Steril 2002;77:776-80.

18. Goswami SK, Das T, Chattopadhyay R, Sawhney V, Kumar J, Chaudhury K, et al. A randomized single-blind controlled trial of letrozole as a low-cost IVF protocol in women with poor ovarian response: a preliminary report. Hum Reprod 2004;19:2031-5.

19. Garcia-Velasco JA, Moreno L, Pacheco A, Guillen A, Duque L, Requena A, et al. The aromatase inhibitor letrozole increases the concentration of intraovarian androgens and improves in vitro fertilization outcome in low responder patients: a pilot study. Fertil Steril 2005;84:82-7.

20. Mitwally MF, Biljan MM, Casper R. Pregnancy outcome after the use of an aromatase inhibitor for ovarian stimulation. Am J Obstet Gynecol 2005;192:381-6.

21. Tiboni GM. Aromatase inhibitors and teratogenesis. Fertil Steril 2004;81:1158-9. 
22. Casper RF. (reply of the author). Aromatase inhibitors and teratogenesis. Fertil Steril 2004;81:1159.

23. Arimidex, patient prescribing information. Visitado 2006 Jun 02. Disponible en: http://www.arimidex. net/article/501449.aspx
24. Tulandi T, Martin J, Al-Fadhli R, Kabli N, Forman $\mathrm{R}$, Hitkari J, et al. Congenital malformations among 911 newborns conceived after infertility treatment with letrozole or clomiphene citrate. Fertil Steril 2006;85:1761-5.

\section{Conflicto de intereses: ninguno declarado.}

